\title{
Antimicrobial Resistance within Host: A Population Dynamics View
}

\author{
Chunji Huang ${ }^{1}$ and Aijun Fan ${ }^{2}$ \\ ${ }^{1}$ Xinqiao Hospital, Third Military Medical University, Chongqing 400037, China \\ ${ }^{2}$ Chongqing Research Center for Information and Automation Technology, Chongqing Academy of Science \& Technology, \\ Chongqing 401123, China
}

Correspondence should be addressed to Aijun Fan; aijun71@163.com

Received 27 December 2013; Accepted 19 January 2014; Published 23 February 2014

Academic Editor: Weiming Wang

Copyright (c) 2014 C. Huang and A. Fan. This is an open access article distributed under the Creative Commons Attribution License, which permits unrestricted use, distribution, and reproduction in any medium, provided the original work is properly cited.

\begin{abstract}
To study the relationship between antimicrobial resistance and the concentration of antibiotics, a competitive population dynamical model is proposed between the susceptible strain and the resistant strain with antibiotic exposure. The strict mathematical analysis is performed, and the results indicate that long-term high strength antibiotic treatment and prevention can induce the extinction of susceptible strain. Thus, the prescribed dose of antibiotics must be strictly controlled during the treatment and prevention of the infections in clinics.
\end{abstract}

\section{Introduction}

It was thought that the war against infectious diseases has been won in the initial stages of the discovery of antibiotics and their widespread introduction [1]. However, during the multiplication process of bacteria, there are high degrees of individuality or phenotypic heterogeneity in populations of genetically identical cells [2-5]. As a result of the cell-to-cell variation, a high probability of the selection of antimicrobial resistance is particularly prone to occur. Thus, the overuse of antibiotic therapy may result in the prevalence of antibioticresistant bacteria and an apparently inexorable advent of a postantibiotic era or a super wicked challenge [6-9]. In fact, antimicrobial resistance has now become an unfolding catastrophe [1] and the new strategy and action plan has been proposed by the Department of Health in the United Kingdom [9].

To extend the life of existing antibiotics, it is necessary to analyze the molecular mechanism of antibiotic resistance and strategize about slowdown and avoid antibiotic resistance during anti-infective therapy. In the process numerous research articles have highlighted that both molecular biology and computational biology, including mathematical modeling, are vitally important methods [5, 10-15]. Especially, recent biological study has confirmed that the signaling nucleotide (p)ppGpp can control bacterial persistence by stochastic induction of toxin-antitoxin activity, and there is a special resistant strain, which can switch into slow growth through the changes of (p)ppGpp level in high antibiotic concentration [5]. However, under different concentrations of antibiotic, the long-term competitive ending between the susceptible strain and the resistant strain remains unknown.

In this paper, based on the above mentioned mechanism of bacterial antibiotic resistance within the host, a competitive population dynamical model is proposed to explore the competitive interactions between the susceptible strain and the resistant strain with antibiotic exposure. The focus is the relationship between antibiotic resistance and the concentration of antibiotics, which may be added to the host by injection, orally, or by transfusion. The organization of this paper is as follows. In the next section, the proposed model is described and the global dynamics is obtained. In Section 3, some numerical simulations are performed. Finally, a brief discussion is given to conclude this work.

\section{Model and Its Dynamical Behaviors}

2.1. Description of the Model. According to the pharmacokinetic, we know that the concentration of the drug within-host 
will tend to be approximately constant after multiple dosing. Thus, it is reasonable to assume that the plasma concentration of the antibiotics is a constant, which is denoted as $S_{0}$. In addition, let $x(t)$ be the number of susceptible strains, and let $y(t)$ be the number of resistant strains at time $t$, respectively. The following differential equations can be used to describe the basic dynamics of the interaction between $x(t)$ and $y(t)$ :

$$
\begin{gathered}
\frac{\mathrm{d} x(t)}{\mathrm{d} t}=x(t)\left(r_{1}-\delta_{11} x(t)-\delta_{12} y(t)-\beta S_{0}\right) \triangleq F_{1}(x, y), \\
\frac{\mathrm{d} y(t)}{\mathrm{d} t}=y(t)\left(r_{2} e^{-\mu S_{0}}-\delta_{21} x(t)-\delta_{22} y(t)\right) \triangleq F_{2}(x, y),
\end{gathered}
$$

where the natural growth rates and death rates of susceptible strain and resistant strain are $r_{1}, r_{2}, \delta_{11} x(t)$, and $\delta_{22} y(t)$, respectively. Parameter $\beta$ is the coefficient of the effect of destroying susceptible bacteria by antibiotics, and function $e^{-\mu S_{0}}$ denotes the decline of growth rate of resistant strain by the signaling nucleotide (p)ppGpp. For biological consistency, all parameters are positive constants, $r_{1}>\beta S_{0}$ and the initial values of system (1) are $x(0)>0$ and $y(0)>0$.

2.2. Mathematical Analysis. Because of the biological meaning of the components $(x(t), y(t))$, we focus on the model in the first octant of $\mathbb{R}^{2}$. To study the dynamics of system (1), we first show that that model (1) is biologically well behaved and dissipative; that is, all solutions of model (1) in $\mathbb{R}_{+}^{2}$ are ultimately bounded and the solutions with positive initial values are positive.

Theorem 1. Under the given initial conditions, all solutions of system (1) are positive and system (1) is dissipative.

This theorem is clear to be seen, thus, the detailed proof is omitted for the sake of simplicity.

In order to obtain the global dynamics of system (1), we first have the following result regarding the nonexistence of periodic orbits in system (1).

Theorem 2. System (1) does not have nontrivial periodic orbits.

Proof. Consider system (1) for $x>0$ and $y>0$. Take a Dulac function:

$$
D(x, y)=\frac{1}{x y}
$$

We have

$$
\frac{\partial D(x, y) F_{1}(x, y)}{\partial x}+\frac{\partial D(x, y) F_{2}(x, y)}{\partial y}=-\frac{\delta_{11}}{y}-\frac{\delta_{22}}{x}<0 .
$$

The conclusion follows from Dulac criterion $[16,17]$.

We now consider the existence of equilibria of system (1). Let $F_{1}(x, y)=0$ and let $F_{2}(x, y)=0$. Clearly, when the plasma concentration of the antibiotics $S_{0}<r_{1} / \beta$, model (1) always has three equilibria: one is $E_{0}=(0,0)$, meaning that both bacteria become extinct and the others are $E_{1}=\left(x_{1}, 0\right)$ and $E_{2}=\left(0, y_{2}\right)$, in which

$$
x_{1}=\frac{r_{1}-\beta S_{0}}{\delta_{11}}, \quad y_{2}=\frac{r_{2} e^{-\mu S_{0}}}{\delta_{22}},
$$

which are corresponding to the extinction of resistant strain and susceptible strain, respectively. Furthermore, we have the positive equilibrium $E_{+}=\left(x^{*}, y^{*}\right)$, corresponding to coexistence of susceptible strain and resistant strain, that is given by intersections of the zero growth isoclines:

$$
\begin{aligned}
& l_{1}: r_{1}-\delta_{11} x(t)-\delta_{12} y(t)-\beta S_{0}=0, \\
& l_{2}: r_{2} e^{-\mu S_{0}}-\delta_{21} x(t)-\delta_{22} y(t)=0 .
\end{aligned}
$$

Apparently, the isoclines $l_{1}$ and $l_{2}$ pass through the points $E_{1},\left(0, y_{1}\right)$ and $\left(x_{2}, 0\right), E_{2}$, respectively. Here

$$
y_{1}=\frac{r_{1}-\beta S_{0}}{\delta_{11}}, \quad x_{2}=\frac{r_{2} e^{-\mu S_{0}}}{\delta_{21}} .
$$

According to the position relation between $l_{1}$ and $l_{2}$, we know that there are four cases (Figure 1) depending on the size of parameters $A_{1}, A_{2}, A_{3}$, and $A_{4}$, in which

$$
\begin{array}{ll}
A_{1}=r_{2} \delta_{12} e^{-\mu S_{0}}+\beta \delta_{22} S_{0}, & A_{2}=r_{1} \delta_{22}, \\
A_{3}=r_{2} \delta_{11} e^{-\mu S_{0}}+\beta \delta_{21} S_{0}, & A_{4}=r_{1} \delta_{21} .
\end{array}
$$

The object of the next analysis is to study the asymptotical stabilizability of the equilibria. Since $l_{1}$ and $l_{2}$ are the isoclines of system (1), $l_{1}$ and $l_{2}$ divide the first octant into several subregions, and the derivative of $x$ and $y$ keeps a fixed sign in each subregion as indicated in Figure 1. By the combination of Theorem 1, Theorem 2, and the Poincaré-Bendixson theorem, with the help of the fixed sign in each subregion (Figure 1), we have the complete dynamical behaviors of system (1), which is summarized in Table 1.

\section{Simulations}

From Table 1, we know that equilibrium $E_{2}$ is globally asymptotically stable in the case of (IV); that is, the susceptible strain will extinct and the resistant strain will persist, which means that antimicrobial resistance occurs. What is the relationship between the concentration of antibiotics and the phenomenon of antimicrobial resistance? In this section, we will give some qualitative analyses from a numerical simulation standpoint.

Let

$$
\begin{array}{llrl}
r_{1}=1.5, & r_{2}=3.5, & \delta_{11}=2.0, \\
\delta_{22}=12.0, & \beta=1.0, & \mu & =0.5 .
\end{array}
$$

When $\delta_{12}=4.0$ and $\delta_{21}=3.0$, if there is no antibiotics, that is, $S_{0}=0$, after a simple calculation, we have $A_{1}<A_{2}$ and $A_{3}>A_{4}$. Thus, Case (I) occurs (Figure 2(a)). Increasing 
TABLE 1: Dynamical behaviors of system (1).

\begin{tabular}{lll}
\hline Case & \multicolumn{1}{c}{ Conditions } & Dynamics \\
\hline I & $A_{1}<A_{2}$ and $A_{3}>A_{4}$ & $E_{0}, E_{1}$, and $E_{2}$ are unstable, and $E_{+}$is globally asymptotically stable \\
II & $A_{1}>A_{2}$ and $A_{3}<A_{4}$ & $E_{0}$ and $E_{+}$are unstable; $E_{1}$ and $E_{2}$ are locally stable dependent on the \\
III & $A_{1}<A_{2}$ and $A_{3}<A_{4}$ & initial conditions \\
IV & $A_{1}>A_{2}$ and $A_{3}>A_{4}$ & $E_{0}$ and $E_{2}$ are unstable, and $E_{1}$ is globally asymptotically stable \\
\hline
\end{tabular}

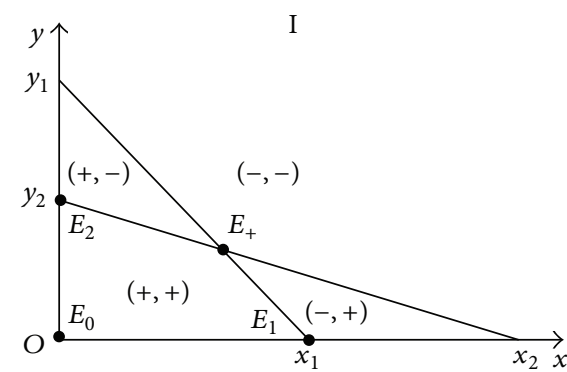

(a)

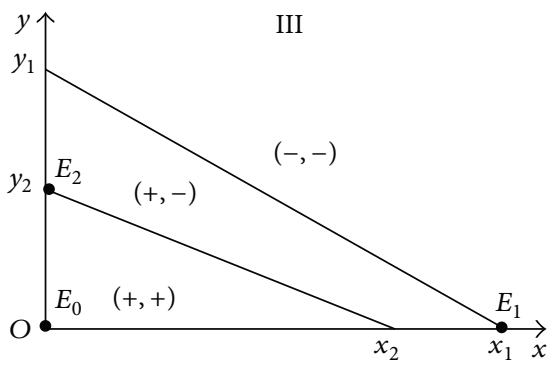

(c)

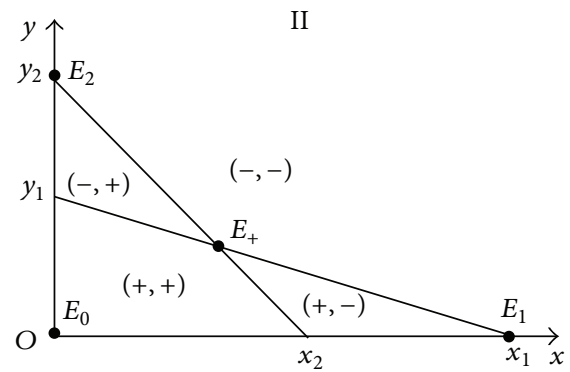

(b)

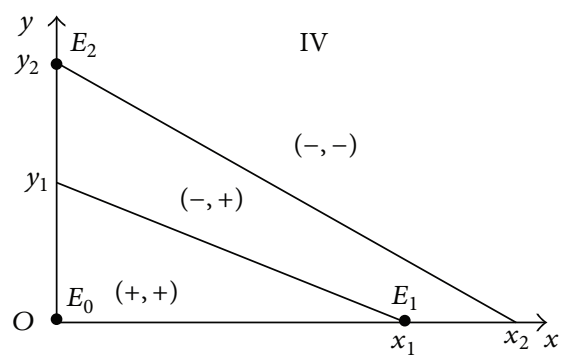

(d)

FIgure 1: Illustration of the equilibria and the vector field of system (1). (I) $A_{1}<A_{2}$ and $A_{3}>A_{4}$; (II) $A_{1}>A_{2}$ and $A_{3}<A_{4}$; (III) $A_{1}<A_{2}$ and $A_{3}<A_{4}$; (IV) $A_{1}>A_{2}$ and $A_{3}>A_{4}$. Each equilibrium is represented by a closed cycle $(\bullet)$, and the sign of the derivative of $x, y$ in each subregion is denoted by $(a, b)$, where $a$ is the sign of the derivative of $x$ and $b$ is the sign of the derivative of $y$. Note that the expressions of $x_{1}, x_{2}, y_{1}, y_{2}, A_{1}, A_{2}, A_{3}$, and $A_{4}$ are shown in (4), (6), and (7).

the concentration of antibiotics, $S_{0}=0.1$, the inequalities remain valid. However, when the concentration of antibiotics increase to $S_{0}=0.9$, we find that the inequalities become $A_{1}>A_{2}, A_{3}>A_{4}$, and Case (IV) occurs, which is also shown in Figure 2(a). Thereby long-term high strength antibiotic treatment and prevention can induce the extinction of susceptible strain and accelerate the phenomenon of antimicrobial resistance.

By changing the parameter $\delta_{21}$ to 6.0 , because $A_{1}<$ $A_{2}$ and $A_{3}<A_{4}$ are valid, we can obtain the extinction of resistant strain and persistence of susceptible strain if there is no antibiotics or low strength antibiotic treatment (Figure 2(b), Case (III) in Table 1). Similarly, when there is a high strength antibiotic treatment, $S_{0}=0.9$, the inequalities change to $A_{1}>A_{2}$ and $A_{3}>A_{4}$ (Case (IV) in Table 1) and the simulated time series is shown in Figure 2(b). Thus, the serious consequences of the abuse of antibiotic were proved afresh during the treatment and prevention of the infections.

Holding $\delta_{21}=6.0$ and changing the parameter $\delta_{12}$ to 5.5, the inequalities $A_{1}>A_{2}$ and $A_{3}<A_{4}$ are valid if $S_{0}=0.0$ or $S_{0}=0.1$ (Case (II) in Table 1). Thus, both extinction and persistence of the resistant strain may happen in course of the competition because $E_{1}$ and $E_{2}$ are locally stable dependent on the initial conditions (Figures 2(c) and 2(d)). However, when the concentration of antibiotics increase to $S_{0}=$ 0.9 , the resistant strain is survived since the inequalities change to $A_{1}>A_{2}$ and $A_{3}>A_{4}$ (Case (IV) in Table 1) and the equilibrium $E_{2}$ is globally asymptotically stable (Figures $2(c)$ and $2(d)$ ), which also means that it is necessary to control the dose of antibiotics. Otherwise, antimicrobial resistance will occur.

\section{Discussion}

According to the latest mechanism of bacterial antibiotic resistance within the host [5], a competitive population model (1) between the susceptible strain and resistant strain is proposed under the circumstance of antibiotic exposure. Based on the global dynamics of system (1), the relationship is explored between antimicrobial resistance and the concentration of antibiotics by numerical simulations. The results indicate that the resistant strain will ultimately survive along with the long-term high strength antibiotic treatment and 


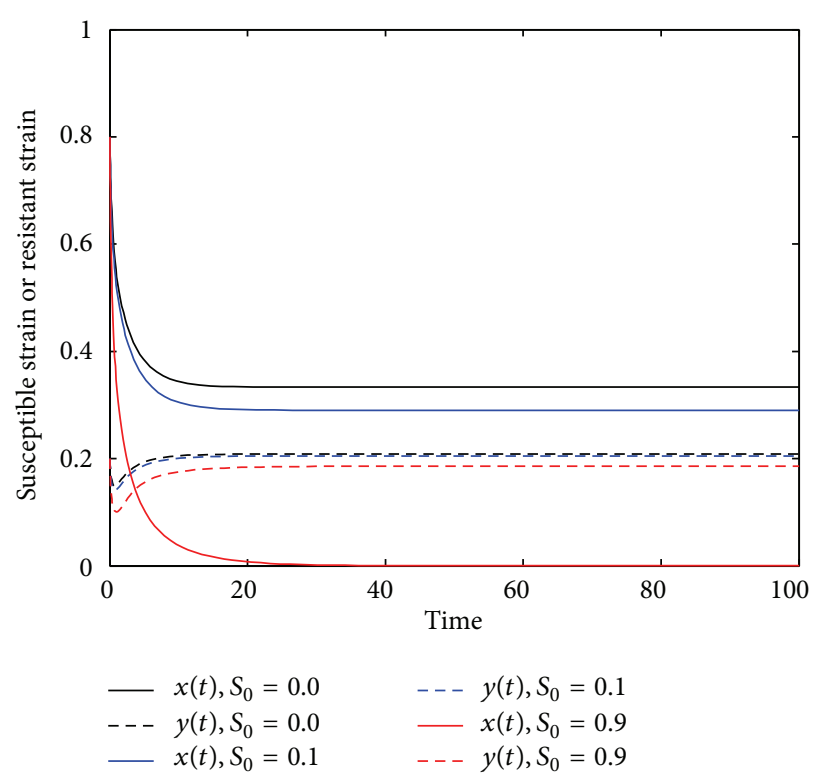

(a)

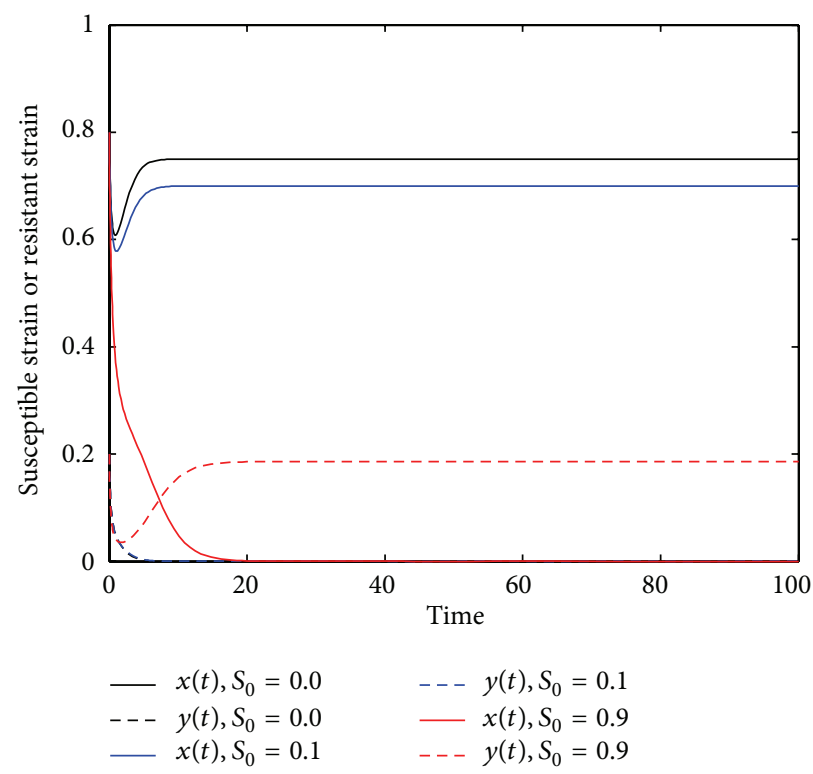

(c)

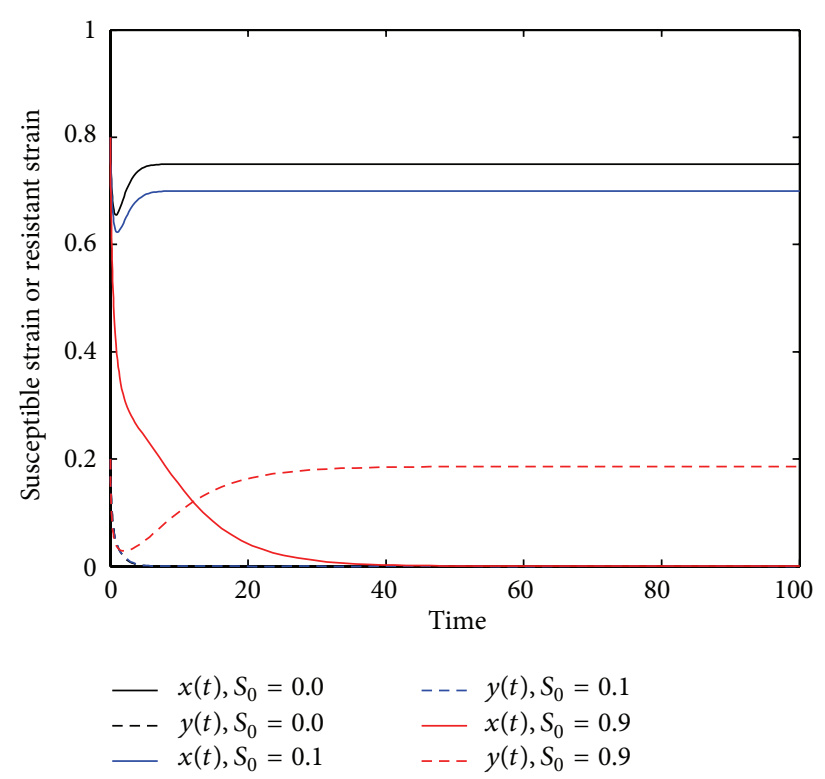

(b)

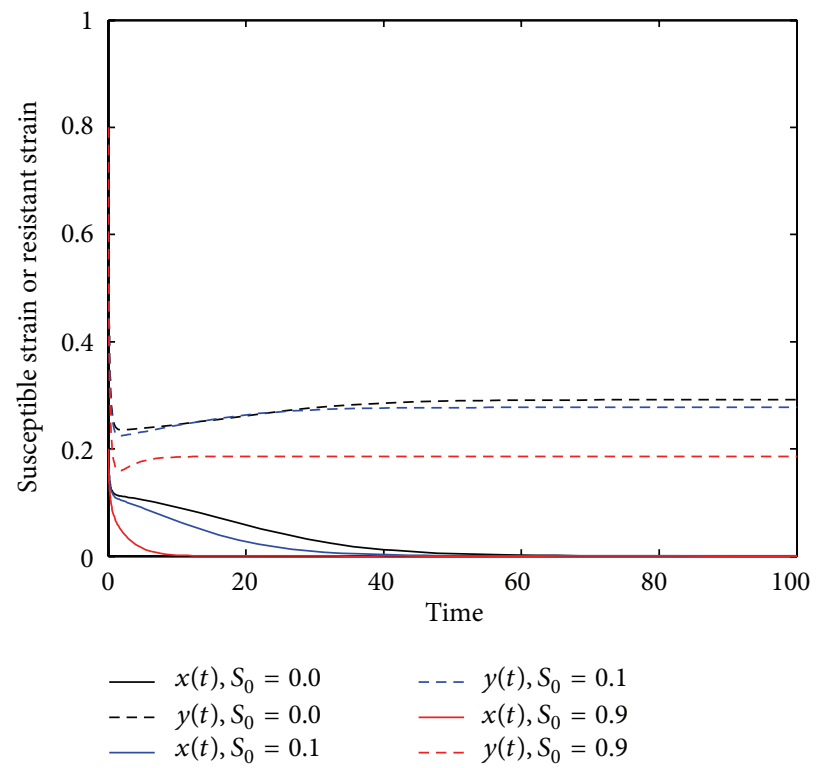

(d)

FIGURE 2: Time series of the susceptible strain $(x(t))$ and resistant strain $(y(t))$ within host as predicted by the model $(1)$. $\delta_{12}=4.0$ and $\delta_{21}=3.0$ in (a); $\delta_{12}=4.0$ and $\delta_{21}=6.0$ in (b); $\delta_{12}=5.5$ and $\delta_{21}=6.0$ in (c) and (d). The initial condition is $(x(0), y(0))=(0.8,0.2)$ in $(\mathrm{a})$, $(\mathrm{b})$, and $(\mathrm{c})$, and $(x(0), y(0))=(0.2,0.8)$ in $(\mathrm{d})$. Other parameters are shown in (8).

prevention, which has been found in many recurrent and chronic infections [18-20].

Note that the assumption that infections can be prevented or treated has become the backbone of the whole modern healthcare [1]. Thus, resistance is not just an infectious disease issue, it is also a surgical issue, a cancer issue, and a health system issue [1]. Antimicrobial prescribing needs to be more evidence based and more efficiently targeted [9]. In particular, in order to inhibit or decelerate resistance to antibiotics, the prescribed dose of antibiotics must be strictly controlled during the treatment and prevention of the infections in clinics. Otherwise, a postantibiotic era or a super wicked challenge is likely to occur [6-9]. Though the risk-benefit balance for antibiotic prescribing is becoming even more complex [9], mathematical modeling may be a useful research tool because it can involve and integrate a wide range of subjects, including biology, medicine, and economics.

\section{Conflict of Interests}

The authors declare that there is no conflict of interests regarding the publication of this paper. 


\section{Acknowledgment}

This work is supported by the Research Institutes Innovation Capacity-Building Program of CQ (no. cstc2012ptkyys40002).

\section{References}

[1] F. Godlee, "Antimicrobial resistance-an unfolding catastrophe," British Medical Journal, vol. 346, article f1663, 2013.

[2] D. Dubnau and R. Losick, "Bistability in bacteria," Molecular Microbiology, vol. 61, no. 3, pp. 564-572, 2006.

[3] A. Eldar and M. B. Elowitz, "Functional roles for noise in genetic circuits," Nature, vol. 467, no. 7312, pp. 167-173, 2010.

[4] M. E. Lidstrom and M. C. Konopka, "The role of physiological heterogeneity in microbial population behavior," Nature Chemical Biology, vol. 6, no. 10, pp. 705-712, 2010.

[5] E. Maisonneuve, M. Castro-Camargo, and K. Gerdes, "(p)ppGpp controls bacterial persistence by stochastic induction of toxin-antitoxin activity," Cell, vol. 154, no. 5, pp. 1140-1150, 2013.

[6] F. E. Berkowitz, "Antibiotic resistance in bacteria," Southern Medical Journal, vol. 88, no. 8, pp. 797-804, 1995.

[7] F. C. Tenover and J. M. Hughes, "The challenges of emerging infectious diseases: development and spread of multiplyresistant bacterial pathogens," Journal of the American Medical Association, vol. 275, no. 4, pp. 300-304, 1996.

[8] A. M. Garber, "Antibiotic exposure and resistance in mixed bacterial populations," Theoretical Population Biology, vol. 32, no. 3, pp. 326-346, 1987.

[9] A. S. Kessel and M. Sharland, "The new UK antimicrobial resistance strategy and action plan," British Medical Journal, vol. 346, article f1601, 2013.

[10] M. Lipsitch, C. T. Bergstrom, and B. R. Levin, "The epidemiology of antibiotic resistance in hospitals: paradoxes and prescriptions," Proceedings of the National Academy of Sciences of the United States of America, vol. 97, no. 4, pp. 1938-1943, 2000.

[11] M. J. M. Bonten, D. J. Austin, and M. Lipsitch, "Understanding the spread of antibiotic resistant pathogens in hospitals: mathematical models as tools for control," Clinical Infectious Diseases, vol. 33, no. 10, pp. 1739-1746, 2001.

[12] N. Jumbe, A. Louie, R. Leary et al., "Application of a mathematical model to prevent in vivo amplification of antibiotic-resistant bacterial populations during therapy," Journal of Clinical Investigation, vol. 112, no. 2, pp. 275-285, 2003.

[13] A. Sotto and J. P. Lavigne, "A mathematical model to guide antibiotic treatment strategies," BMC Medicine, vol. 10, article 90, 2012.

[14] P. Ankomah and B. R. Levin, "Two-drug antimicrobial chemotherapy: a mathematical model and experiments with Mycobacterium marinum," PLoS Pathogens, vol. 8, no. 1, Article ID e1002487, 2012.

[15] I. H. Spicknall, B. Foxman, C. F. Marrs, and J. N. S. Eisenberg, "A modeling framework for the evolution and spread of antibiotic resistance: literature review and model categorization," American Journal of Epidemiology, vol. 178, no. 4, pp. 508-520, 2013.

[16] C. C. McCluskey and J. S. Muldowney, "Bendixson-Dulac criteria for difference equations," Journal of Dynamics and Differential Equations, vol. 10, no. 4, pp. 567-575, 1998.
[17] O. Osuna and G. Villasenor, "On the Dulac functions," Qualitative Theory of Dynamical Systems, vol. 10, no. 1, pp. 43-49, 2011.

[18] K. Lewis, "Persister cells, dormancy and infectious disease," Nature Reviews Microbiology, vol. 5, no. 1, pp. 48-56, 2007.

[19] M. D. LaFleur, Q. Qi, and K. Lewis, "Patients with longterm oral carriage harbor high-persister mutants of Candida albicans," Antimicrobial Agents and Chemotherapy, vol. 54, no. 1, pp. 39-44, 2010.

[20] K. R. Allison, M. P. Brynildsen, and J. J. Collins, "Metaboliteenabled eradication of bacterial persisters by aminoglycosides," Nature, vol. 473, no. 7346, pp. 216-220, 2011. 


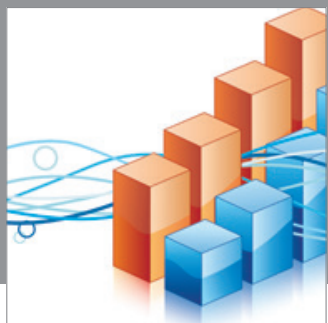

Advances in

Operations Research

mansans

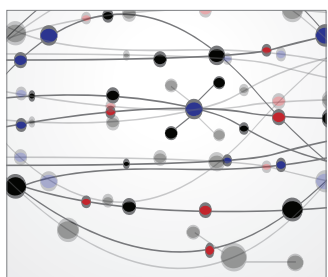

The Scientific World Journal
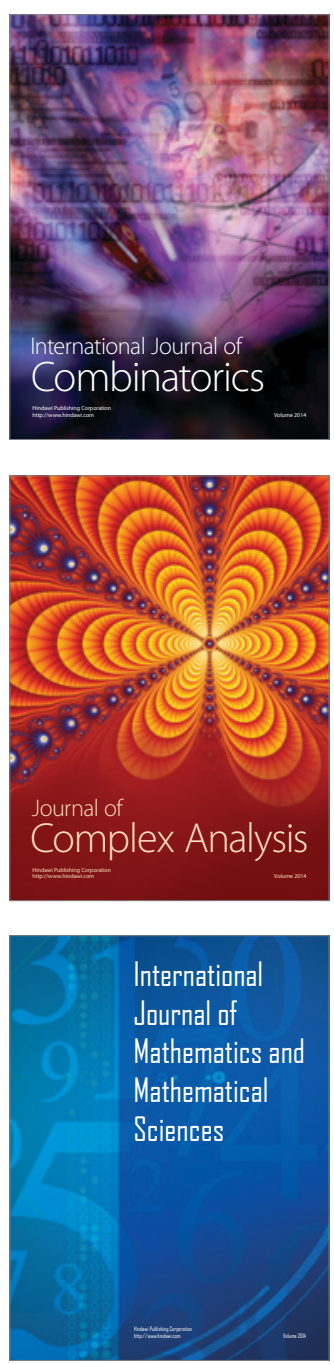
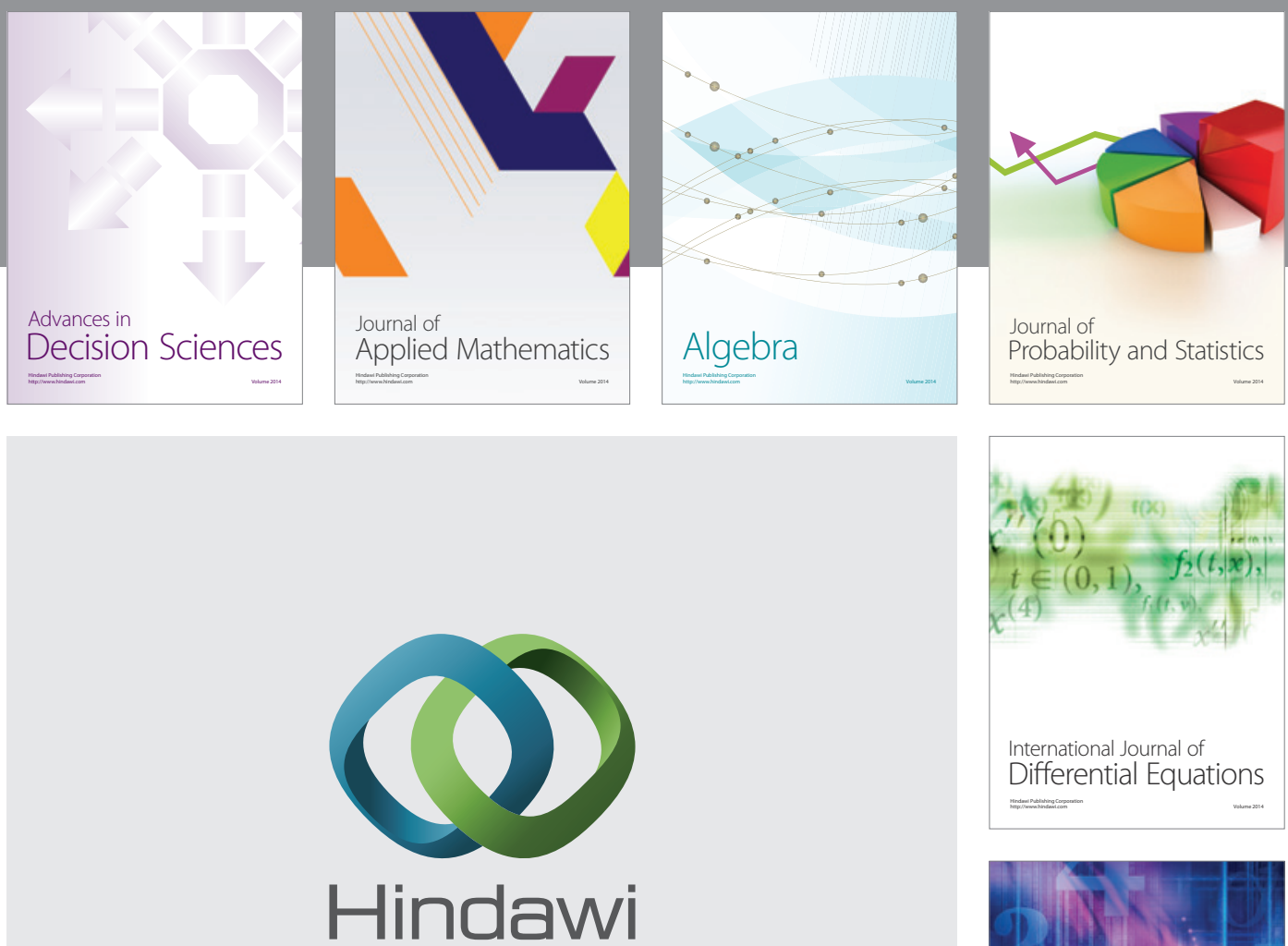

Submit your manuscripts at http://www.hindawi.com
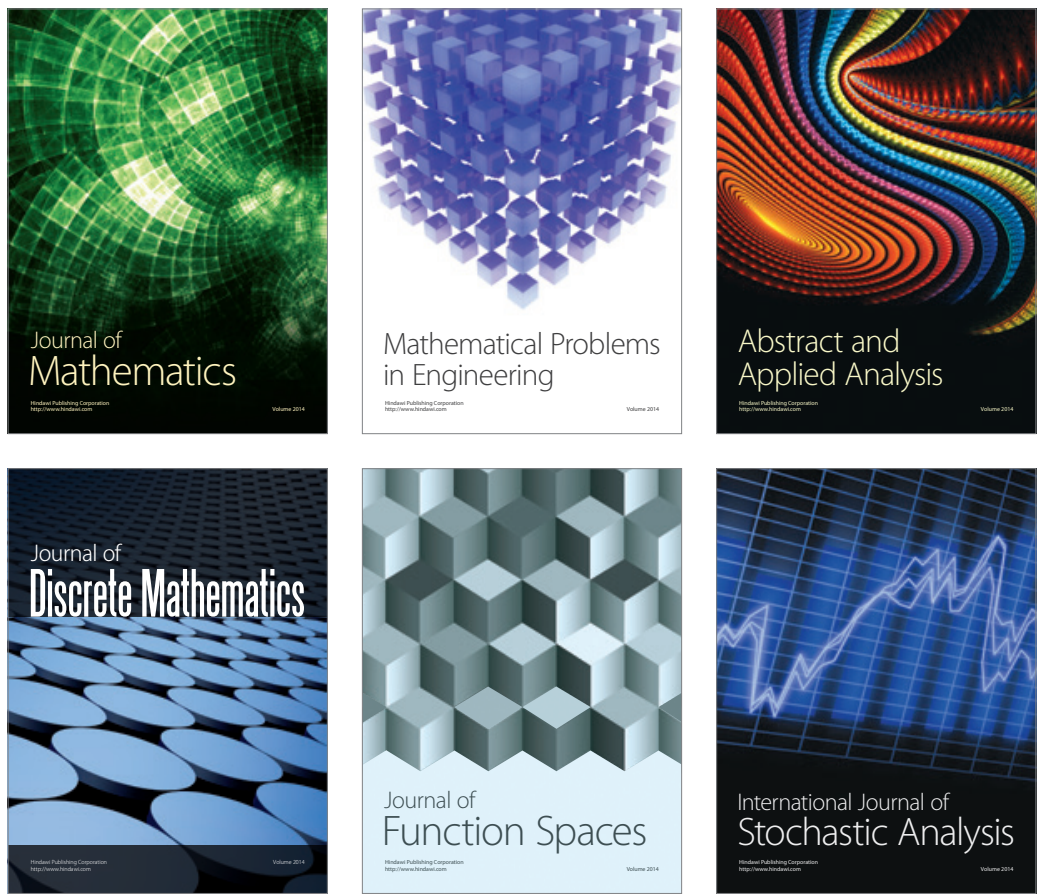

Journal of

Function Spaces

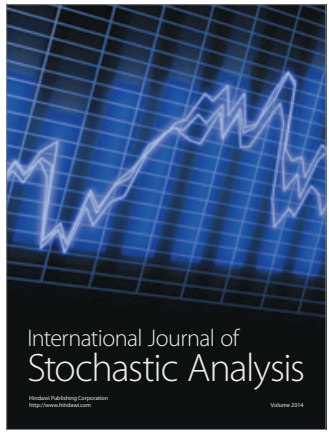

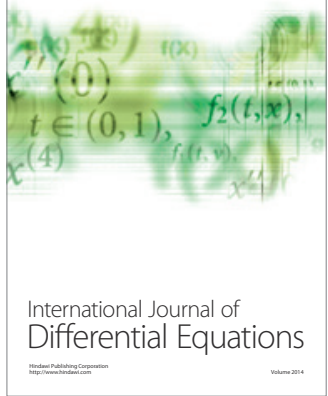
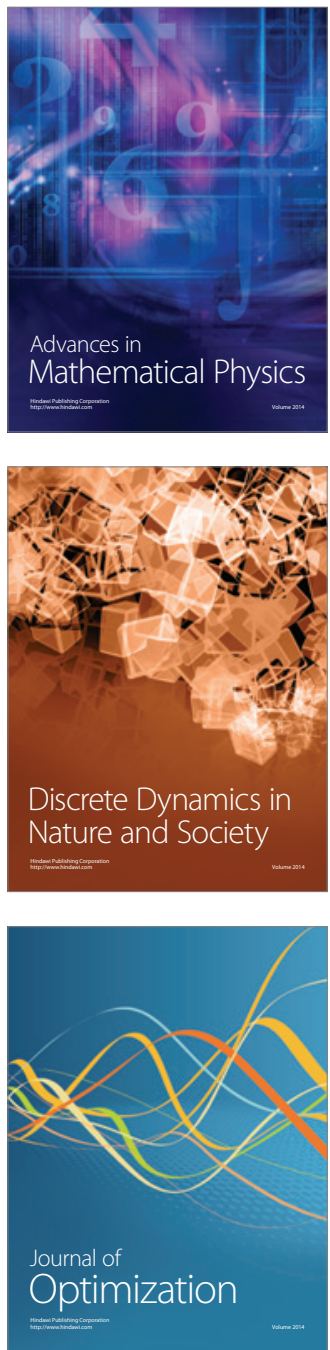